Article

\title{
Four Novel Cellulose Synthase (CESA) Genes from Birch (Betula platyphylla Suk.) Involved in Primary and Secondary Cell Wall Biosynthesis
}

\author{
Xuemei Liu ${ }^{1}$, Qiuyu Wang ${ }^{1,2}$, Pengfei Chen ${ }^{1}$, Funan Song ${ }^{1}$, Minxiao Guan ${ }^{1}$, Lihua Jin ${ }^{1}$, \\ Yucheng Wang ${ }^{1,2, *}$ and Chuanping Yang ${ }^{1,2, *}$
}

1 Northeast Forestry University, 26 Hexing Road Xiangfang District, Harbin 150040, China; E-Mails: liuxuemei@nefu.edu.cn (X.L.); wqyll@sina.com (Q.W.); cpfnefu1234@sohu.com (P.C.); sfn@nefu.edu.cn (F.S.); gmx.06@163.com (M.G.); 1hjin2000@hotmail.com (L.J.)

2 State Key Laboratory of Forest Genetics and Tree Breeding, Northeast Forestry University, 26 Hexing Road, Harbin 150040, China

* Authors to whom correspondence should be addressed; E-Mails: ychngwang@yahoo.com (Y.W.); yangchuanpingnefu@yahoo.com (C.Y.); Tel.: +86-451-8219-0607 (ext. 12) (Y.W.); Fax: +86-451-8219-0607 (ext. 11) (Y.W.).

Received: 6 June 2012; in revised form: 7 September 2012 / Accepted: 11 September 2012 / Published: 25 September 2012

\begin{abstract}
Cellulose synthase (CESA), which is an essential catalyst for the generation of plant cell wall biomass, is mainly encoded by the $\operatorname{Ces} A$ gene family that contains ten or more members. In this study; four full-length cDNAs encoding CESA were isolated from Betula platyphylla Suk., which is an important timber species, using RT-PCR combined with the RACE method and were named as BplCesA3, $-4,-7$ and -8 . These deduced CESAs contained the same typical domains and regions as their Arabidopsis homologs. The cDNA lengths differed among these four genes, as did the locations of the various protein domains inferred from the deduced amino acid sequences, which shared amino acid sequence identities ranging from only $63.8 \%$ to $70.5 \%$. Real-time RT-PCR showed that all four BplCesAs were expressed at different levels in diverse tissues. Results indicated that BplCESA8 might be involved in secondary cell wall biosynthesis and floral development. BplCESA3 appeared in a unique expression pattern and was possibly involved in primary cell wall biosynthesis and seed development; it might also be related to the homogalacturonan synthesis. BplCESA7 and BplCESA4 may be related to the formation of a cellulose synthase complex and participate mainly in secondary cell wall biosynthesis. The
\end{abstract}


extremely low expression abundance of the four BplCESAs in mature pollen suggested very little involvement of them in mature pollen formation in Betula. The distinct expression pattern of the four BplCesAs suggested they might participate in developments of various tissues and that they are possibly controlled by distinct mechanisms in Betula.

Keywords: birch; CESA; primary cell wall; secondary cell wall; transcript; wood; cellulose; gene expression

Abbreviations: CESA: Cellulose synthase; cDNA: Complementary DNA; EST: Expressed Sequence Tag; NCBI: National Center for Biotechnology Information; ORF: Open Reading Frame; CDD: the Conserved Domain Database; TMDs: Transmembrane Domains; HVR: Hypervariable Regions; CRP: the Plant Conserved Region; QRT-PCR: Quantitative Reverse Transcription Polymerase Chain Reaction; RACE: Rapid Amplification of cDNA ends; UDP: Uridine 5'-diphosphate; Csl: CESA-like; CSR: the Class-Specific Region; pI: Isoelectric Point; TMHMM: Transmembrane helix prediction; DEPC: Diethylpyrocarbonate; CTAB: Cetyltrimethyl Ammonium Bromide.

\section{Introduction}

Cellulose, a key structural component of the plant cell wall, is the most abundant biopolymer in the world. Cellulose is a homopolymer consisting of $\beta$-1,4-glucan chains that are synthesized at the plasma membrane by membrane-localized "rosette" complexes [1], as visualized by freeze-fracture electron microscopy [2-5]. To date, cellulose synthase (CESA) has been localized in these cellulose-synthesizing complexes. CESA plays a central role in plant cell wall biomass formation [1,6]. Each plant synthesizes a number of different cellulose synthases and each cellulose-synthesizing complex contains at least three non-redundant cellulose synthase isoforms. Both genetic and biochemical evidence suggest that different CESA isoforms interact to form a functional cellulose synthase enzyme complex [7]. CESA uses UDP-glucose as a substrate and polymerizes glucose residues in a single-step reaction [2]. Ces $A$ genes are part of a CesA/CSL superfamily, and the proteins they encode contain the same domains and regions as CESA proteins of coniferous gymnosperms [8], angiosperms and molds [9].

Most higher plants express two contrasting groups of apparently co-regulated CesAs involved in either primary or secondary cell wall biosynthesis. The Arabidopsis genome encodes 10 CesA genes belonging to six classes known to participate in cellulose microfibril biosynthesis [10]. Expression of at least three different gene products, AtCESA1, AtCESA3 and one of the CESA6-related CESAs (AtCESA2, AtCESA5, AtCESA6 or AtCESA9), are required for primary cell wall formation. AtCESA4, AtCESA7 and AtCESA8 are required for the development of the thick secondary wall [11-13]. In rice, 45 sequences that significantly matched the CesA/CSL superfamily were revealed by searching the TIGR database, of which 11 were predicted as OsCesA. OsCesA1, -3 , and -8 showed high co-expression in the tissues of the primary cell wall, whereas $O s C e s A 4,-7$, and -9 were co-expressed in the secondary cell wall tissues [14]. In corn, three of the 12 CesA genes are involved in cellulose synthesis during secondary wall formation $[2,15]$. Trees are perennial species that accumulate massive 
amounts of secondary xylem (i.e., wood) [16]. Within the CesA family of some tree species, complexes exist that are engaged in the deposition of cellulose during primary cell wall synthesis and secondary cell wall formation similar to Arabidopsis and rice. In aspen (Populus tremuloides Mickx.), PtdCesA1 [17], PtdCesA2 [18] and PtdCesA3 [19] are associated with secondary cell wall development. PtdCesA4, PtdCesA5, PtdCesA6 and PtdCesA7 are involved in primary cell wall development in aspen trees [20-22]. Here, it should be noted that all CesAs in Populus were renamed according to the nomenclature for the cellulose synthase genes in $P$. trichocarpa proposed by Kumar et al. in 2009 [23]. Microarray profiling in P. trichocarpa has demonstrated that PtiCesA3-C, 4, 6-C, 7-A, 8-A and 8-B were clearly xylem-specific, while remaining PtiCesA having detectable transcript molecules did not exhibit clear tissue specificity. PtiCesA4, PtiCesA7-A, $-B$ and PtiCesA8-A, -B are the homologs of Arabidopsis AtCesA4, AtCesA7 and AtCesA8, respectively [24]. The three AtCesA genes are required for the biosynthesis of cellulose in the secondary cell walls [11-13]. Gene expression analysis by QRT-PCR in Eucalyptus, another tree species of great commercial interest, indicated that transcripts of $E g C e s A 1$ to 3 were related to development of secondary cell walls [25]. Expression of EgCesA4 and EgCesA5 was related to primary wall synthesis, whereas EgCesA6 was weakly expressed in all tissues [25]. Furthermore, eight CesA genes were identified in the coniferous gymnosperm Pinus radiata [8]. Nairn and Haselkorn [26] described three secondary cell wall-associated CesAs from loblolly pine gymnosperm (Pinus taeda L.), while recent research reported 10 CesAs in Pinus taeda although there was little evidence for their involvement in cell wall biosynthesis [27].

Betula platyphylla Suk., a fibrous, broadleaf commercial tree species widely distributed in the northeast of China, has many applications in architecture, furniture and paper production. However, some important economic characteristics of many tree species, such as the quality and properties of the wood, are controlled by multiple genes with undetermined genetic mechanisms. Thus, understanding the mechanism of cellulose formation would enable the improvement of fiber characteristics and the creation of new birch cultivars using biotechnology. We isolated four members of the CesA family from B. platyphylla and identified the structural elements of their deduced protein sequences. The tissue-specific expression patterns of these genes were compared at different developmental stages in Betula.

\section{Results}

\subsection{Cloning the CesA Genes from B. platyphylla}

Four full-length candidate CesA cDNA sequences were isolated from leaves and stems of B. platyphylla. A search for conserved domains or functional motifs in the CDD revealed that the four encoded proteins possessed two separate conserved domains: a glycosyltransferase domain $\left(E\right.$ value $\left.=2 \times 10^{-11}\right)$ and a cellulose synthase domain, which are typical of CESAs $\left(E\right.$ value $\left.<1.0 \times 10^{-180}\right)[2,4]$. Therefore, the four genes were confirmed as $C e s A$ s and designated BplCes $A 3,-4,-7$ and -8 , using the three-letter prefix nomenclature $(B p l)$ for cellulose synthase genes reported in Populus [23]. 


\subsection{Structure and Properties of BplCESAs of B. platyphylla}

The four full-length CesAs ranged from 3255 to 3968 bp in length, containing ORF lengths ranging from 2958 to $3255 \mathrm{bp}$, encoding 985 to 1084 amino acids (Table 1). The four putative BplCESAs differed at residues 11 to 33 of the $N$ terminus and 16 residues at the $\mathrm{C}$ terminus (Figure 1) and shared only $63.8 \%$ to $70.5 \%$ identity (Table 1 ), which was similar to that shared by the seven aspen CESAs $(64 \%-76 \%)$ [18,22]. However, the putative BplCESAs shared the highest identity $(85 \%-98 \%)$ with orthologs in other plants. Importantly, the four BplCESAs contained all the typical motifs of plant cellulose synthases described by Joshi [19], including the plant conserved region (CRP), eight predicted transmembrane regions, a conserved zinc finger motif $\left(\mathrm{CX}_{2} \mathrm{CX} n \mathrm{CX}_{2} \mathrm{CX} n \mathrm{CX}_{2} \mathrm{C}\right)$ (Figure 2) and the conserved D, D, D, QxxRW signature sequence (D, D, D, QVLRW) involved in substrate binding and catalysis during cellulose synthesis (Figure 1). The predicted plant-specific conserved and hypervariable regions (HVRs) shared only approximately $16 \%$ to $35 \%$ identity. Thus, all these genes are predicted to be true CesA genes rather than cellulose synthase-like (CSL) genes as defined by Richmond and Somerville [10].

Table 1. Properties and main domains of four deduced BpCESA proteins.

\begin{tabular}{|c|c|c|c|c|c|}
\hline \multirow{2}{*}{ Property } & \multirow{2}{*}{ Symbol } & \multicolumn{4}{|c|}{ Location in deduced protein sequence } \\
\hline & & BpCESA8 & BpCESA3 & BpCESA7 & BpCESA4 \\
\hline Genbank accession & Genbank ACC & EU591529 & EU591530 & EU591531 & EU591532 \\
\hline Full lengh (bp) & & 3255 & 3968 & 3399 & 3457 \\
\hline \multirow{2}{*}{ Untranslated region } & 5'-UTR & 40 & 419 & 23 & 53 \\
\hline & 3'-UTR & 257 & 293 & 236 & 257 \\
\hline Open reading frame (bp) & ORF & 2958 & 3255 & 3123 & 3147 \\
\hline Number of amino acid & aa & 985 & 1084 & 1040 & 1048 \\
\hline Isoelectric point & $\mathrm{p} I$ & 6.27 & 6.84 & 5.90 & 6.74 \\
\hline \multirow[t]{4}{*}{ Molecular weight } & MW (kDa) & 110.4 & 121.2 & 117.7 & 119.5 \\
\hline & TMD1 & $176-192$ & $275-291$ & $252-268$ & $227-251$ \\
\hline & TMD2 & $203-219$ & $302-318$ & $272-299$ & $253-273$ \\
\hline & TMD3 & $765-783$ & $855-882$ & $814-840$ & $830-847$ \\
\hline \multirow{5}{*}{$\begin{array}{l}\text { transmembrane domain } \\
\text { (TMDs) }\end{array}$} & TMD4 & $794-812$ & 895-912 & $850-869$ & $859-877$ \\
\hline & TMD5 & $829-848$ & $929-955$ & $886-912$ & 894-919 \\
\hline & TMD6 & $878-900$ & $981-1003$ & $937-958$ & $945-965$ \\
\hline & TMD7 & $911-932$ & $1015-1033$ & $971-989$ & $976-997$ \\
\hline & TMD8 & $943-960$ & $1047-1063$ & $1003-1019$ & $1007-1026$ \\
\hline $\mathrm{Zn}$ finger $(\mathrm{Zn}) \mathrm{CxxC}$ & Zinc finger $46 \mathrm{bp}$ & $9-54$ & $20-65$ & $37-82$ & $41-86$ \\
\hline \multirow{2}{*}{ hypervariable region (HVR) } & HVRI & $55-174$ & $66-273$ & $83-250$ & $87-224$ \\
\hline & HVRII & $545-669$ & $644-770$ & $620-726$ & $594-735$ \\
\hline \multirow[t]{2}{*}{ plant conserved region (CRP) } & CRP 125bp & $301-425$ & $400-524$ & $377-501$ & $351-474$ \\
\hline & U1 16bp & $285-300$ & $384-399$ & $361-376$ & $335-350$ \\
\hline \multirow{4}{*}{$\begin{array}{l}\text { UDP-glucose binding and } \\
\text { catalysis sites }(\mathrm{U})\end{array}$} & $\mathrm{U} 218 \mathrm{bp}$ & $455-472$ & $554-571$ & $530-547$ & $504-521$ \\
\hline & $\mathrm{U} 322 \mathrm{bp}$ & $678-699$ & $779-800$ & $735-756$ & $744-765$ \\
\hline & $\mathrm{U} 4$ 18bp & $716-733$ & $817-834$ & $773-790$ & $782-799$ \\
\hline & BpCESA 8 & 1 & 64.4 & 63.8 & 66.6 \\
\hline \multirow[t]{2}{*}{$\%$ Similarity to } & BpCESA3 & / & / & 70.5 & 65.6 \\
\hline & BpCESA7 & l & 1 & 1 & 67.3 \\
\hline
\end{tabular}


Figure 1. Multiple sequence alignment and main domains for the deduced amino acid sequences of four CESA proteins. Shown are the conserved processive glycosyltransferase motif (D, D, D, QVLRW, black triangles), zinc finger motif, hypervariable regions (HVRI and HVRII) and the plant conserved region (CRP). Black shading indicates amino acid identities.

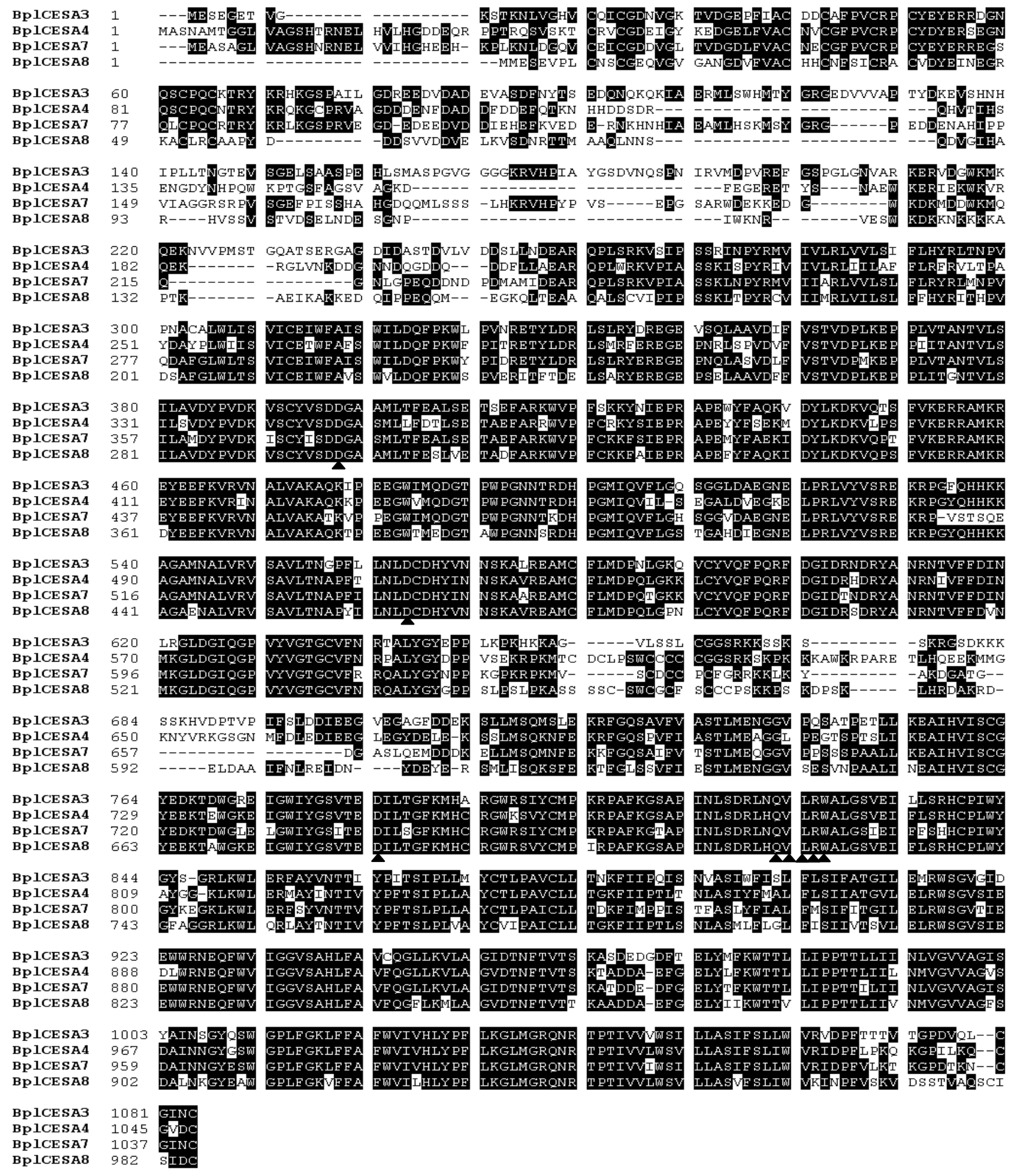


Figure 2. Diagrammatic representation of the four deduced CESA amino acid sequences of Betula. Blue, zinc finger region; black, eight transmembrane domains; green, CRP domain; red, HVRI and HVRII/CSR domains; light blue, motifs necessary for glycosyl transferase processivity [D, D, D, QXXRW (U1-U4)].

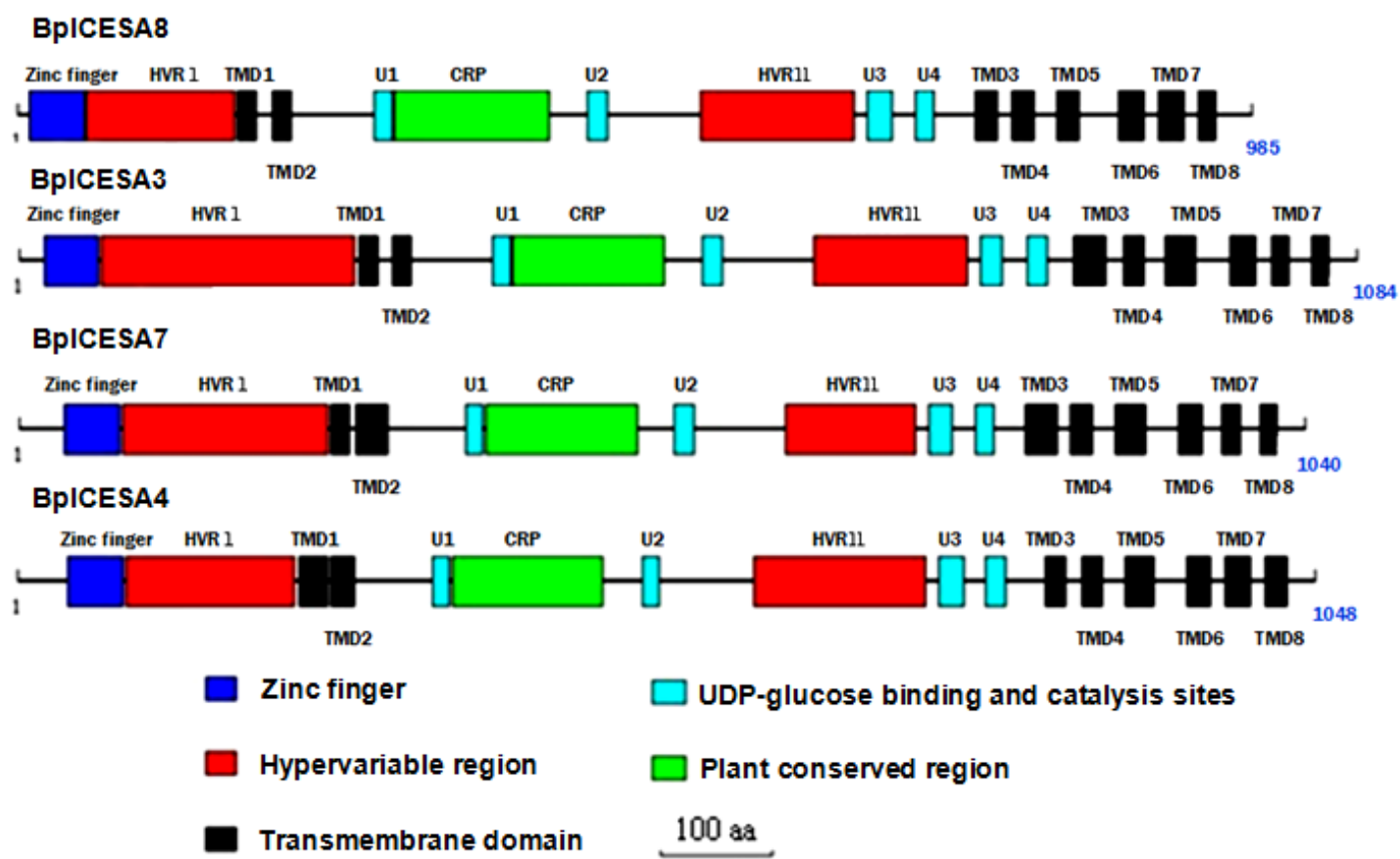

\subsection{Phylogenetic Analysis of BplCesA Sequences}

It is estimated that the Populus genome has $18 \operatorname{Ces} A$ genes [24,28,29], which can be grouped into eight sets of clearly defined paralogs, plus a single copy of PtiCesA4 [23,28]. We used the 39 predicted full-lengh CESA amino acid sequences from five plant species, Physcomitrella patens (PpCESA8 as outgroup), Arabidopsis thaliana (10 AtCESAs), P. trichocarpa (17 PtiCESAs), P. tremuloides (7 PtdCESAs) and B. platyphylla (4 BplCESAs) to generate a phylogenetic tree (Figure 3 ). The multiple sequence alignment was shown in Figure S1. The two hypervariable regions (HVRI and HVRII) of the selected CesA gene products were highly divergent and excluded for the construction of the phylogenetic tree. The four Betula CESAs clustered with specific Arabidopsis and Populus CESA homologs ( $>80 \%$ identity) to form discrete clades (Figure 3). For example, BplCESA8 clustered with PtiCESA8-A/PtiCESA8-B (88.8\%-89.2\% amino acid sequence identity), AtCESA8 (86.6\%) and PtdCESA1 (87.4\%). BplCESA3 was considered orthologous to the PtiCESA3-C/PtiCESA3-D paralogs (92.8\%-93.1\% identity), while AtCESA3 (91.3\%) and PtdCESA5 (89.6\%). BplCESA7 shared $89.7 \%$ to $89.3 \%$ identity with PtiCESA7-A/PtiCESA7-B, $85.0 \%$ with AtCESA7 and $89.2 \%$ with PtdCESA2. BplCESA4 clustered with the single set comprising PtiCESA4 (89.9\% identity), AtCESA4 (86.1\%) and PtdCESA3 (88.0\%). Genes in such clusters presumably share a common evolutionary history in which an ancestral CESA existed before the divergence of Betula, Arabidopsis and Populus. This parallel numbering model suggests that the clustered CESAs share similar functions in the three species. In addition, three predicted protein sequences of the four BplCESAs shared a maximum identity of $97.8 \%$ to $99.2 \%$ with three CESA isoforms from $B$. luminifera, whereas the other two CESA homologs, BplCESA3 and BluCESA5, shared only $63.6 \%$ to $71.9 \%$ identity with other 
CESAs of the two Betula species (Data not shown). Further investigations are required to confirm that the diverged CesA genes in Betula species perform distinct roles in cellulose biosynthesis.

Figure 3. Phylogenetic relationships of deduced Arabidopsis; Populus and Betula CESA proteins. To identify the species of origin for each CESA, a species name or acronym is included before the name of the sequences: Pp: Physcomitrella patens; At; Arabidopsis thaliana; Bpl; Betula platyphylla (by arrow); Ptd; Populus tremuloides; Pti; Populus trichocarpa. The GenBank accession numbers are as follows: PpCESA8 (DQ902549); AtCESA1 (At4g32410); AtCESA2 (At4g39350); AtCESA3 (At5g05170); AtCESA4 (At5g44030); AtCESA5 (At5g09870). AtCESA6 (At5g64740); AtCESA7 (At5g17420); AtCESA8 (At4g18780); AtCESA9 (At2g21770); AtCESA10 (At2g25540); BplCESA8 (EU591529); BplCESA3 (EU591530); BplCESA7 (EU591531); BplCESA4 (EU591532); PtdCESA1 (AF072131); PtdCESA2 (AY095297); PtdCESA3 (AF527387); PtdCESA4 (AY162181); PtdCESA5 (AY055724); PtdCESA6 (AY196961); PtdCESA7 (AY162180); PtiCESA1-A (Pti835809); PtiCESA1-B (Pti763479); PtiCESA3-A (Pti560520); PtiCESA3-B (Pti576348); PtiCESA3-C (Pti821409); PtiCESA3-D (Pti706420); PtiCESA4 (Pti553321); PtiCESA6-A (Pti207792); PtiCESA6-B (Pti819877); PtiCESA6-C (Pti818594); PtiCESA6-D (Pti551308); PtiCESA6-E (Pti806784); PtiCESA6-F (Pti784751); PtiCESA7-A (Pti717644); PtiCESA7-B (Pti262611); PtiCESA8-A (Pti235238); PtiCESA8-B (Pti555650).

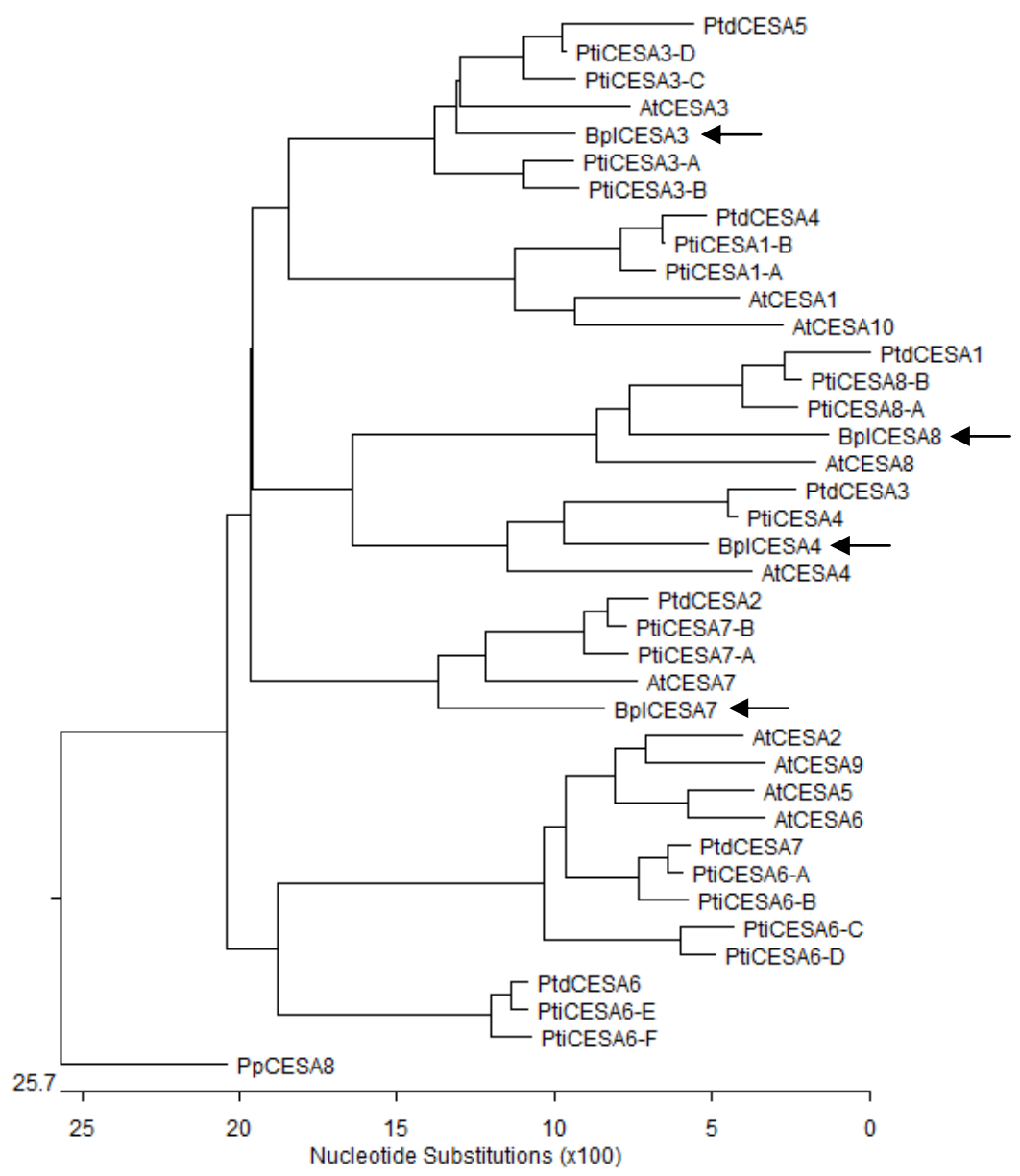




\subsection{Real-Time RT-PCR Analysis of the Expression Profiles of BplCesAs}

In order to research the transcript abundance and characteristics of the four novel BplCesAs in cell wall formation in distinct tissues, transcript expression profiles were generated in 11 different tissues of Betula by quantitative RT-PCR using gene-specific primers (Table 2). In our study, the abundance of all BplCesA mRNAs was detected and normalized to constitutively expressed ACTIN mRNA which has been improved to be qualified as a reference gene by Chao Dai et al. [30]. The results showed that BplCesA 8 was highly expressed in mature tissue, including some tissues with secondary thickening, such as male and female inflorescences (FI and MI), mature petioles as well as young and mid-development stem tissues rich in secondary cell wall (Figure 4). BplCesA3 was predominantly expressed in tissues either typical of primary cell walls (young leaves in mid July) (Figure 4) or consistent with the expression pattern in early development (in July) of leaves as shown in Figure 5a and seeds (Figure 4). BplCesA7 and BplCesA4 were strictly co-expressed and expressed predominantly in developing and mature vascular tissues or tissues with secondary thickening, such as young and mature stems and male inflorescences. Another interesting result is that the four BplCesAs were expressed in mature pollen at an extremely low level compared with other tissues (Figure 4).

Table 2. Degenerate primers and specific primers for isolation and quantitative real-time PCR of BplCesAs.

\begin{tabular}{lll}
\hline Primers & Primer $\mathbf{5}^{\prime} \rightarrow \mathbf{3}^{\prime}$ & Tm $\left({ }^{\circ} \mathbf{C}\right)$ \\
\hline$U 1$ & 5'-TGGATTYTGGATCAGTTCCC-3' & \\
$U 2$ & 5'-TGGATTYTDGATCAGTTCCC-3' & \\
D1 & 5'-TTVCCRAANAGMGGACCCCA-3' & \\
D2 & 5'-CCCATSAGACCYTTGAGGAA-3' & \\
BplactinR & TCA AGT TCC TGC TCA TAG TCA A & 55.3 \\
BplactinF & TTG CTA TCC AGG CTG TTC TC & 55.3 \\
BplCesA8R & TGC TCC ATA CGA TGA CGA CT & 56.0 \\
BplCesA8F & CCT TCC ATC TGC TGC TCT G & 56.1 \\
BplCesA3R & TGT CTG CTG CAT CAC CTG A & 55.5 \\
BplCesA3F & AAA GAG TCA TCC ACA AGC ACA T & 56.4 \\
BplCesA7R & GTA ATA GCC GGT GGT AGA TCC & 55.9 \\
BplCesA7F & TGC TCG AAG CAAT CGG TA & 55.5 \\
BplCesA4R & AGG CAG CAT GTC ACT ATC CA & 55.3 \\
BplCesA4F & TTC TTG CCT GAC TTT CCA CTT C & 56.5 \\
\hline
\end{tabular}

In addition, expression profiles for the four BplCesAs in developing leaves and stems were determined by quantitative RT-PCR using the same gene-specific primers (Table 2). Expression of BplCes $A$ in stems was high during most developmental periods, with abundant expression of BplCesA7 and BplCesA4 (Figure 5). With the exception of BplCesA3, all BplCesAs were expressed at higher levels in stems than in leaves at most stages (Figure 5). BplCesA 8 mRNA was more abundant in stems than in leaves at earlier developmental stages, whereas BplCesA3 mRNA was notably more abundant in leaves, which are richer in primary cell wall than stems at some time-points, especially earlier and later development stages (in July and September) (Figure 5). Both the BplCesA7 and BplCesA4 
mRNAs were more abundant in stems (which are rich in secondary cell walls) than in leaves (which are typical of primary cell walls) during most of the examined periods (Figures 5).

Figure 4. Quantitative real-time RT-PCR analysis of four CesA genes in tissues of B. platyphylla. The mRNA levels are expressed relative to the amount of Bplactin mRNA. FI: female inflorescence, MI: male inflorescence, FS: flower stalk, YL: young leaves, YP: young petiole, YS: young stem, ML: mid-development leaves, MP: mid-development petiole, MS: mid-development stem, SE: seed, PO: mature pollen. Data represent the mean \pm standard error.

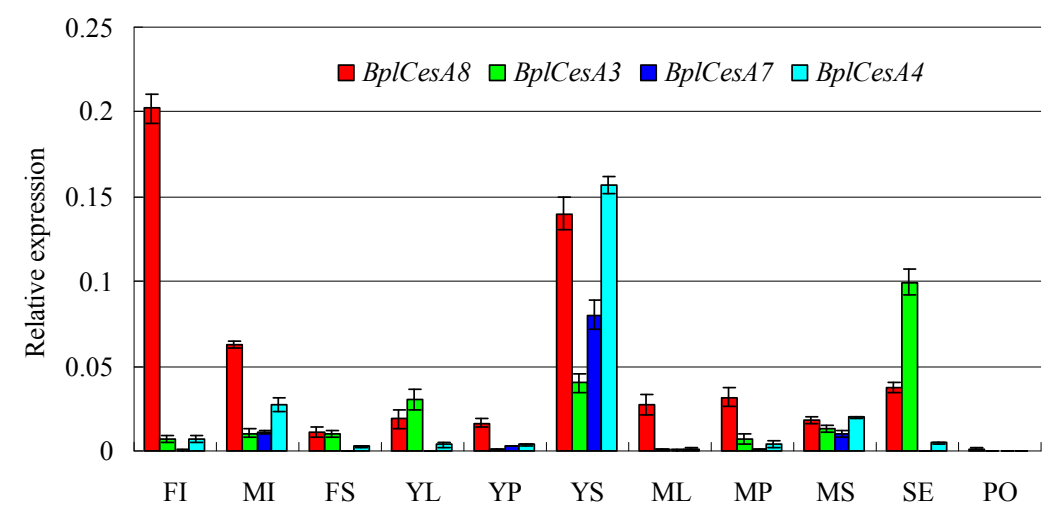

Figure 5. Quantitative real-time RT-PCR analysis of four CesA genes in leaves and stems at different developmental stages in B. platyphylla. The mRNA levels are normalized by Bplactin mRNA. Shown are individual mRNA abundances of the four CesA genes in leaves or stems. Data represent the mean \pm standard error.
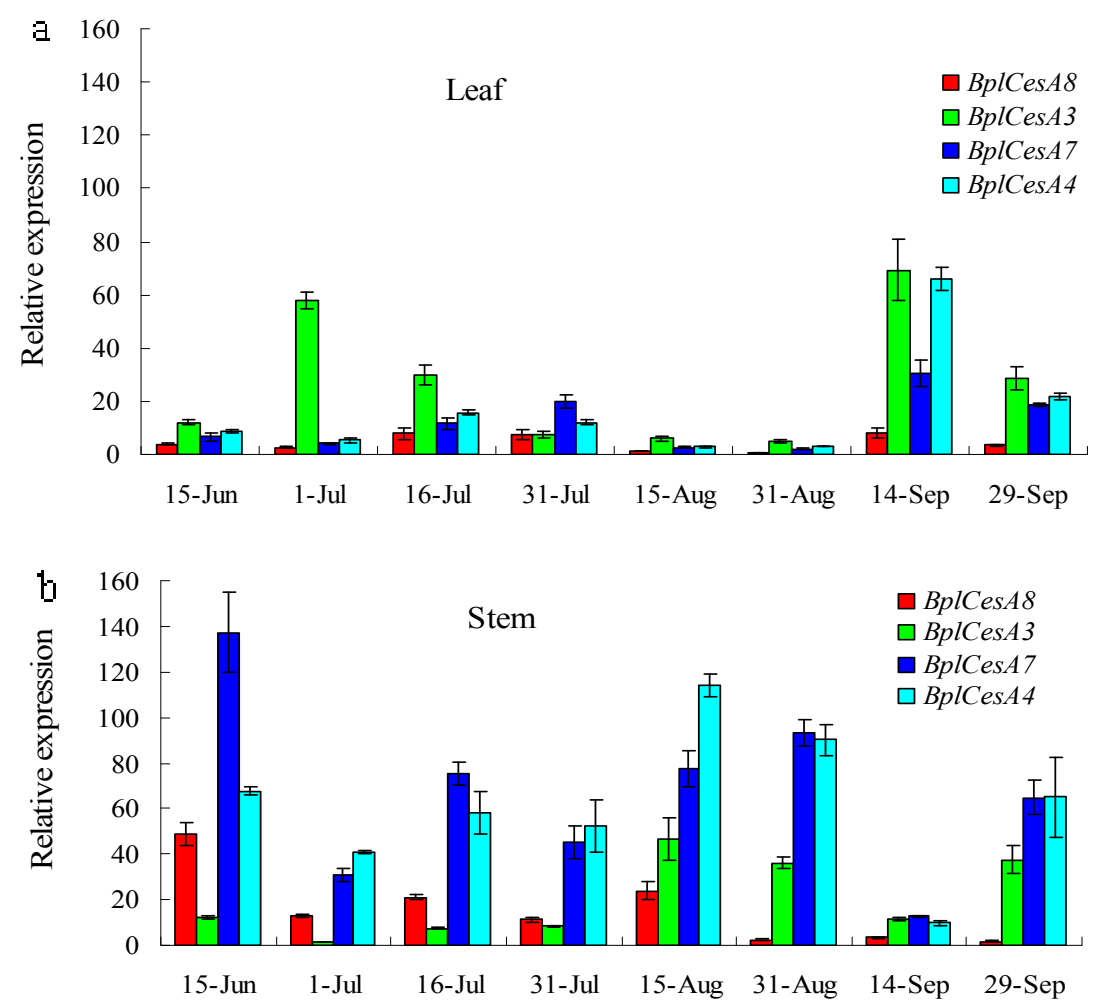


\section{Discussion}

\subsection{The CesA Family of Betula Comprises at Least Four Member Genes}

No other CESA homologs have been identified in B. platyphylla in this study or elsewhere, indicating that the CesA gene family of B. platyphylla is comprised of at least four genes. It has been reported that the CesA gene family is comprised of 10 or more homologs in plants. For example, 93 cellulose synthesis-related genes have been identified in the P. trichocarpa genome, containing 17 CesA genes [23,24,28], 10 CESA homologs in Arabidopsis [12,23], and 11 CesA genes in rice [14]. There are 12 CesA gene members in Pinus [27]. The CesA gene family contains 11 CesA members in the moss (Physcomitrella patens) [31]. Here, only four BplCesAs were identified, which may be due to very low mRNA levels of other BplCesAs in certain tissues or developmental stages. Further studies are required to identify other $B p l C e s A$ s in distinct tissues.

\subsection{The Four BplCesAs Differentially Participate in the Development of Diverse Tissues in Betula}

Each of the CESAs may have specific roles related to the formation of primary and secondary walls in plants. The distinct transcript expression profiles of the four BplCesAs indicated their different involvement in growth of Betula. Almost all of the four BplCesAs were highly expressed in the tissues we examined, indicating their involvement in the biosynthesis of cellulose which is the main component of plant cell walls. BplCesA 8 was more highly expressed in earlier growth stages of the stem (Figure 5) and there were more abundances of BplCesA 8 transcript in some tissues with secondary thickening (female and male inflorescences with abundant bracts and some mature tissues) or undergoing active secondary cell wall formation (in earlier growth as young stems) than other members (Figure 4). This indicated similarity to its homolog AtCESA8, which participates in secondary cell wall biosynthesis in Arabidopsis [11-13]. Similarly, PtiCESA8-A and PtiCESA8-B, which clustered with BplCESA8, also exhibited apparently higher xylem specificity [24]. This indicates BplCESA8 might be involved in secondary cell wall biosynthesis.

Notably we found high transcript expression levels of BplCesA8 in male and female inflorescences (Figure 4). In Arabidopsis, CESA1 appears to be possibly required for either embryo or male gametophyte growth $[32,33]$. CESA3 is coexpressed with CESA1, and homozygous cesa3 alleles are also male gametophyte lethal [13]. By comparison, in rice, OsCESA2,-9 were highly expressed in young panicle and the OsCSLF genes (OSCSLF2 \& -7) were preferentially expressed in the hull of rice, while several CSL (cellulose synthase-like) genes (CSLG2,3 and CSLB2 in sepals, CSLG1, CSLD6 and CSLA1,2,10,11 in Carpels) are specifically expressed in flower organs in Arabidopsis [14]. The homologs of BplCESA8 were not reported to be related to the floral development either in rice or in Arabidopsis, suggesting BplCesA 8 may participate in floral growth in Betula unlike the other two plant species. Despite the involvement of BplCesA 8 in floral growth, there is no evidence for its involvement in male or female gametophyte growth because male and female inflorescences contain abundant bracts.

Also, we could assume BplCesA8 to be a partially redundant candidate gene with BplCesA3. $B p l C e s A 8$ was detected with high transcript levels in specific tissues (male and female inflorescences, young stems) where the expression of BplCesA3 is relatively low, while BplCesA8 was lowly 
expressed in seed where BplCesA3 showed much higher expression (Figure 4). In other words, BplCesA3 may be partially redundant with BplCesA8 in those specific tissues. Furthermore, the expression level of BplCesA8 in female inflorescences very rich in bracts is much higher than that of BplCesA3, while BplCesA3 was much more highly expressed than BplCesA8 in developing seeds which were isolated from female inflorescences (Figure 4). The bracts in inflorescences are typical of secondary thickening cell walls. Thus, BplCesA 8 might be involved in the development of bracts and be related to secondary cell wall formation in Betula as with the result mentioned above.

Both BplCesA7 and BplCesA4 were expressed at apparently higher levels in the stem at most time-points, which are rich in secondary cell walls (Figures 4 and 5), indicating that the two genes may be involved in the biosynthesis of the secondary cell wall throughout the development of the stem. In addition, they were also more highly expressed in old leaves (in September) than young leaves. It is proposed that the changes in expression may reflect a role in the synthesis of homogalacturonan, which accumulates to a high level in old leaves [14]. But in Arabidopsis, it is cellulose synthase-like (CSL) genes (AtCSLD2 and AtCSLE1) which show sequence similarity to CESA apparently exhibiting strong increases in expression in old leaves versus young leaves instead of CesA genes [34]. This suggests the differences in both transcript expression and specific importance in different tissues of the members in the CES/CSL superfamily in the plant. More importantly, BplCesA7 and BplCesA4 were strictly co-expressed. OsCESA4, -7 (homologs of BplCESA4, -7 , respectively) and -9 in rice are thought to be organized as a cellulose synthase complex involved in secondary cell wall synthesis [14], suggesting that the BplCESA7 and BplCESA4 may be related to the formation of a cellulose synthase complex for secondary cell wall biosynthesis. Similarly, the secondary cell wall development-related genes PtdCESA1, PtdCESA2 and PtdCESA3 in P. tremuloides [17-19] are all homologs of BplCESA8, BplCESA7, BplCESA4, respectively (Figure 3), suggesting that these three genes might be associated with secondary cell wall development in Betula.This is also consistent with the xylem-specific expression pattern of PtiCESA7-A or PtiCESA7-B (clustered with BplCESA7) and PtiCESA4 (clustered with BplCESA4) (Figure 3) in P. trichocarpa [24]. Redundant xylem-specific expression may suggest their involvement in the massive production of cellulose in xylem secondary cell walls for wood formation [25]. However, it has been reported that CesAs related to secondary cell walls are not functionally redundant, which suggests that AtCESA4 (a homolog of BplCESA4), AtCESA7 (of BplCESA7) and AtCESA8 (of BplCESA8) are the only CESAs involved in cellulose synthesis and form a complex in the secondary cell wall in Arabidopsis [35]. In spite of this, there is insufficient evidence in this research to support the participation of BplCESA8 (homolog of AtCESA8) complexed with BplCESA7 and BplCESA 4 (homologs of AtCESA7 and AtCESA4, respectively) for secondary cell wall formation.

BplCesA3 exhibited a unique and complex expression pattern (Figures 4 and 5). Although this gene was expressed at lower levels in most tissues compared with the other three BplCesAs, higher expression was detected at the earlier development in young leaves rich in primary cell wall (Figure 4 and Figure 5a). OsCESA1, -3 (homolog of BplCESA3), $-5,-6,-8$ in rice and AtCESA1, $-2,-3$ (homolog of BplCESA3), $-5,-6$ were detected at higher transcript expression levels in young seedlings mainly comprised of young leaves and stem. Also, OsCESA5/OsCESA6 is likely to be partially redundant with OsCESA3 for OsCESA complex organization in the young tissues, such as plumule and radicle [14]. AtCESA3 was reported to function predominantly in the primary cell wall. 
Furthermore, its homolog PtdCESA5 in P. tremuloides is involved in primary cell wall development together with PtdCESA4, PtdCESA6 and PtdCESA7 [21,22]. Usually, three or four CESAs are responsible for primary cell wall biosynthesis in higher plants. AtCESA1 [36], AtCESA 3 and AtCESA6 in Arabidopsis, OsCESA1, OsCESA3 and OsCESA8 in rice [14] and PtdCESA4, PtdCESA5, PtdCESA6 and PtdCESA7 in P. tremuloides [21,22] may form a cellulose synthase complex for primary cell wall biosynthesis in the three species. Therefore, it can be speculated that other primary cell wall related CESAs exist in Betula in addition to BplCESA3. In addition, the higher expression level in the late-development stem reflects a potential relation to the formation of secondary cell wall in Betula. Finally, BplCesA3 was observed at highest expression level in old leaves (Figure 5a), which indicated that BplCesA3 may be related to the synthesis of homogalacturonan similar to BplCesA4, -7 in Betula.

Moreover, the transcript level of BplCesA3 in developing seed was notably higher than in other tissues examined (Figure 4). During the development of seed, the secondary cell walls in the seed protect the embryo. Cellulose is proposed to play a major role in reinforcing the secondary cell wall in the seed coat epidermal cells [37] and to be a component of seed mucilage [38,39]. In Arabidopsis thaliana, AtCESA9 is required for normal secondary wall synthesis in epidermal seed coat cells [37]. AtCESA2, AtCESA5, and AtCESA9 subunits contribute to secondary wall synthesis in epidermal seed coat cells. AtCESA2 and AtCESA9 serve in radial wall reinforcement, as does CESA5, but CESA5 also functions in mucilage biosynthesis in the seed coat epidermis and is indispensable for mucilage attachment to the seed coat These data suggest unique roles for different CESA subunits in one cell type [40]. Though the homolog of BplCESA3, AtCESA3, has not been reported about its participation in the development of seed, AtCESA4, $-7,-8,-9,-10$ were detected highly expression levels in seed and silique [14]. The high transcript level of BplCesA3 in developing seed reflects its possible involvement in development of seed in Betula. To sum up, the unique expression pattern of BplCesA3 mentioned above indicated that it is possibly participating in diverse developments in Betula compared with the other three CESAs examined. Also, BplCESA3 might possess different transcript expression mechanisms in development of Betula from other plants.

We also found an unexpected result that almost each of the four $B p l C e s A 3,-4,-7$ and -8 was observed at extremely low transcript level in mature pollen in Betula (Figure 4). It has been reported that AtCESA2, AtCESA6 and AtCESA9 are coexpressed during pollen development [11]. Triple cesa2cesa6cesa9 mutant plants are pollen lethal, indicating that they function redundantly in pollen development. CESA9 has been proved to function redundantly with CESA6 during pollen development [13]. The extremely low transcript abundances of the four BplCesAs reflected their lower involvement in mature pollen development and that there would be other CesAs related to the maturity of pollen in Betula.

\section{Materials and Methods}

\subsection{RT-PCR and Rapid Amplification of cDNA Ends (RACE) of Four CesAs}

Young and mature leaves and stem tissues (after bark removal, the cambium and stem tissues were scraped with clean blades free of RNase contamination) were harvested from annual branches of 
mature birch trees growing in forests under natural conditions in the Northeast of China. Total RNA was extracted using the CTAB method and was treated with RNase-free DNase I (Promega, USA) to remove DNA contamination. The cDNA was synthesized from $2 \mu \mathrm{g}$ total RNA using Oligo(dT) as the reverse primer in the reverse transcription-PCR (RT-PCR) system (Invitrogen, USA). The cDNA was used as a template for amplification in $50 \mu \mathrm{L}$ PCR buffer (TaKaRa, China). Two upstream (U1 and U2) and two downstream (D1 and D2) degenerate primers (Table 2) were designed with Primer Premier 5.0 software based on conserved motif sequences of eight CESAs mRNAs from other plants [Populus tremuloides CesA2 (AY095297), Populus trichocarpa CesA6-E (Pti806784), Eucalyptus grandis CesA3 (DQ014507), Arabidopsis thaliana CesA3 (At5g05170), Bambusa oldhamii CesA5 (DQ020213), Pinus taeda CesA3 (AY789652), Pinus radiata CesA1 (AY639654), Zea mays CesA12 (AY372246)]. PCR products were separated by agarose gel electrophoresis, and amplified products (approximately 2000-bp) were isolated and ligated into the pGEM-T easy vector (Promega, USA). 5'- and 3'-RACE were carried out with the SMART RACE cDNA amplification kit (Clontech, USA) employing gene-specific primers inferred from the PCR fragments (Table 2). The full-length sequences of BplCesAs were submitted to GenBank with the accession numbers EU591529, EU591530, EU591531 and EU591532.

\subsection{Sequence Alignments and Phylogenetic Reconstruction}

Open reading frames were identified by the ORF Finder tool [41,42]. Putative protein sequences were aligned using Clustal W2 with default parameters (BioEdit version 7.0.0.0; Tom Hall: Raleigh, NC, USA, 2005) [43] and MEGA 4.0 [44]. The alignments were edited with the BioEdit program. Transmembrane domains were identified and analyzed by TMpred [45]. The Populus genome assembly version $1.1[29,46]$ was searched for CESA homologs. The phylogenetic tree was constructed based on the CLUSTAL W algorithm of MegAlign (version 5.01; DNAStar: Madison, WI, USA, 2001). For statistical analysis, 100 bootstrap replications were analyzed. The two hypervariable regions (HVRI and HVRII) of the selected CesA gene products were highly divergent and excluded for the construction of the multiple sequence alignment and phylogenetic tree. The largest part of the sequences containing the conserved cellulose synthase domain was selected to generate the tree, including CRP, eight predicted transmembrane regions, a conserved zinc finger motif and the conserved D, D, D, QxxRW signature sequence involved in substrate binding and catalysis during cellulose synthesis. Domain conservation was assessed by the conserved domain database (CDD Version 2.18; National Center for Biotechnology Information: Bethesda, MD, USA) in the NCBI website $[47,48]$.

\subsection{Real-Time RT-PCR Analysis}

Real-time RT-PCR was carried out on RNA derived from young and old leaves and stems (eight developmental stages) of three 20-year-old mature B. platyphylla grown under natural conditions in the Northeast of China in 2007. Each sample was comprised of a pool of identical quantities of RNA from three birch plants. The cDNA corresponding to the HVRs (hypervariable regions) can be used as gene-specific primers or probes for molecular analyses [18,49,50]. Therefore, specific primer pairs (18-23 bp) (Table 2) were designed based on the two HVRs of the four BplCesA 
mRNAs and the specificity of the primers was tested by RT-PCR (Figure S2) and sequencing (Data not shown). The B. platyphylla Actin gene (GenBank accession number: EU588981) [51] was used as an internal control (reference gene) to normalize the amount of total RNA present in each reaction, which has been improved by Chao Dai et al. in 2011 [30]. The validity of the Bplactin gene as a control gene has been tested and proved by pre-experiment before the real-time RT-PCR (Figure S2). Experiments were carried out on a MJ OpticonTM2 machine (Bio-Rad, Hercules, CA) using the QuantiTect SYBR-green PCR Master Mix (TOYOBO, Osaka, Japan). All reactions were carried out in triplicate for technical and biological repetitions. PCR was performed in a $20 \mu \mathrm{L}$ mixture consisting of

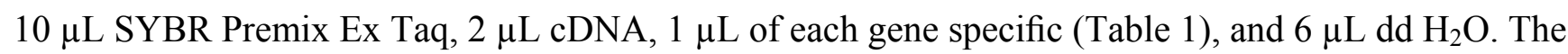
PCR amplification conditions were $95{ }^{\circ} \mathrm{C}$ for $5 \mathrm{~min}$, followed by 40 cycles at $95{ }^{\circ} \mathrm{C}$ for $30 \mathrm{~s}, 60{ }^{\circ} \mathrm{C}$ for $30 \mathrm{~s}, 72{ }^{\circ} \mathrm{C}$ for $30 \mathrm{~s}, 79{ }^{\circ} \mathrm{C}$ for $1 \mathrm{~s}$. Data from qRT-PCR experiments were analyzed by relative quantification according to [52]. The mRNA levels were normalized by the reference gene Bplactin mRNA. A melting curve was generated for each sample at the end of each run to assess the purity of the amplified products.

\section{Conclusions}

In this study, four members of the cellulose synthase (CESA) gene family were isolated and identified from a broadleaf tree species, Betula platyphylla Suk. The four putative BplCESA amino acid sequences contained the typical signature of the most processive glycosyltransferases and exhibited sequence identities ranging from $63.8 \%$ to $70.5 \%$. Furthermore, the transcriptional expression level of the four $B p l C e s A$ s was detected in diverse tissues and developmental periods were found to result in differential expression patterns. Results indicated that BplCesA8 is related to secondary cell wall biosynthesis and floral development. BplCesA3 is predominantly involved in primary cell wall biosynthesis and seed development, and also may be related to the synthesis of homogalacturonan, while BplCESA4 and BplCESA7 appear to participate in secondary cell wall biosynthesis and may be involved together in the formation of a cellulose synthase complex. These data indicate that these genes might be differentially involved in cellulose synthesis, cell wall development and diverse development in tissues in Betula. Further investigations are required to identify other $B p l C e s A$ gene members and their biological functions in Betula.

\section{Acknowledgments}

This work was financially supported by grants from the National High Technology Research and Development Program of China (863 Program) (2011AA100202), the National Natural Science Foundation of China (31100449) and the Fundamental Research Funds for the Central Universities (DL09DAQ01).

\section{References}

1. Kimura, S.; Laosinchai, W.; Itoh, T.; Cui, X.; Linder, C.R.; Brown, R.M. Immunogold labeling of rosette terminal cellulose-synthesizing complexes in the vascular plant Vigna angularis. Plant Cell 1999, 11, 2075-2085. 
2. Bohnert, H.; Nguyen, H.; Lewis, N.G. Bioengineering and Molecular Biology of Plant Pathways; Science Press: Beijing, China, 2009; pp. 136-155.

3. Doblin, M.S.; Kurek, I.; Jacob-Wilk, D.; Delmer, D.P. Cellulose biosynthesis in plants: From genes to rosettes. Plant Cell Physiol. 2002, 43, 1407-1420.

4. Mutwil, M.; Debolt, S.; Persson, S. Cellulose synthesis: A complex complex. Curr. Opin. Plant Biol. 2008, 11, 252-257.

5. Paredez, A.R.; Somerville, C.R.; Ehrhardt, D.W. Visualization of cellulose synthase demonstrates functional association with microtubules. Science 2006, 312, 1491-1495.

6. Schober, M.S.; Burton, R.A.; Shirley, N.J. Analysis of the (1;3)-b-D-glucan synthase gene family of barley. Phytochemistry 2009, 70, 713-720.

7. Perrin, R.M. Cellulose: How many cellulose synthases to make a plant? Curr. Biol. 2001, 11, R213-R216.

8. Krauskopf, E.; Harris, P.J.; Putterill, J. The cellulose synthase gene PrCESA10 is involved in cellulose biosynthesis in developing tracheids of the gymnosperm Pinus radiate. Gene 2005, 350, 107-116.

9. Fugelstad, J.; Bouzenzana, J.; Djerbi, S.; Guerriero, G.; Ezcurra, I.; Teeri, T.; Arvestadd, L.; Bulone, V. Identification of the cellulose synthase genes from the Oomycete Saprolegnia monoica and effect of cellulose synthesis inhibitors on gene expression and enzyme activity. Fungal Genet. Biol. 2009, 46, 759-767.

10. Richmond, T.A.; Somerville, C.R. The cellulose synthase superfamily. Plant Physiol. 2000, 124, 495-498.

11. Taylor, N.G.; Howells, R.M.; Huttly, A.K.; Vickers, K.; Turner, S.R. Interactions among three distinct CesA proteins essential for cellulose synthesis. Proc. Natl. Acad. Sci. USA 2003, 100, 1450-1455.

12. Desprez, T.; Juraniec, M.; Crowell, E.F.; Jouy, H.; Pochylova, Z.; Parcy, F.; Hörte, H.; Gonneau, M.; Vernhettes, S. Organization of cellulose synthase complexes involved in primary cell wall synthesis in Arabidopsis thaliana. Proc. Natl. Acad. Sci. USA 2007, 104, 15572-15577.

13. Persson, S.; Paredez, A.; Carroll, A.; Palsdottir, H.; Doblin, M.; Poindexter, P.; Khitrov, N.; Auer, M.; Somerville, C.R. Genetic evidence for three unique components in primary cell-wall cellulose synthase complexes in Arabidopsis. Proc. Natl. Acad. Sci. USA 2007, 104, 15566-15571.

14. Wang, L.Q.; Guo, K.; Li, Y.; Tu, Y.Y.; Hu, H.Z.; Wang, B.R.; Cui, X.C.; Peng, L.C. Expression profiling and integrative analysis of the CESA/CSL superfamily in rice BMC. Plant Biol. 2010, $10,282$.

15. Appenzeller, L.; Doblin, M.; Barreiro, R.; Wang, H.; Niu, X.M.; Kollipara, K.; Carrigan, L.; Tomes, D.; Chapman, M.; Dhugga, K.S. Cellulose synthesis in maize: Isolation and expression anlysis of the cellulose synthase (CesA) gene family. Cellulose 2004, 11, 287-299.

16. Mellerowicz, E.J.; Baucher, M.; Sundberg, B.; Boerjan, W. Unravelling cell wall formation in the woody dicot stem. Plant Mol. Biol. 2001, 47, 239-274.

17. Wu, L.; Joshi, C.P.; Chiang, V.L. A xylem-specific cellulose synthase gene from aspen (Populus tremuloides) is responsive to mechanical stress. Plant J. 2000, 22, 495-502. 
18. Samuga, A.; Joshi, C.P. A new cellulose synthase gene (PtrCesA2) from aspen xylem is orthologous to Arabidopsis AtCesA7(irx3)gene associated with secondary cell wall synthesis. Gene 2002, 296, 37-44.

19. Joshi, C.P. Xylem-specific and tension stress responsive expression of cellulose synthase genes from aspen trees. Appl. Biochem. Biotechnol. 2003, 105-108, 17-25.

20. Joshi, C.P.; Bhandari, S.; Ranjan, P.; Kalluri, U.C.; Liang, X.; Fujino, T.; Samuga, A. Genomics of cellulose biosynthesis in poplars. New Phytol. 2004, 164, 53-61.

21. Kalluri, U.C.; Joshi, C.P. Differential expression patterns of two cellulose synthase genes are associated with primary and secondary cell wall development in aspen trees. Planta 2004, 220, $47-55$.

22. Samuga, A.; Joshi, C.P. Differential expression patterns of two new primary cell wall-related cellulose synthase cDNAs; PtrCesA6 and PtrCesA7 from aspen trees. Gene 2004, 334, 73-82.

23. Kumar, M.; Thammannagowda, S.; Bulone, V.; Chiang, V.; Han, K.H.; Joshi, C.P.; Mansfield, S.D.; Mellerowicz, E.; Sundberg, B.; Teeri, T.; et al. An update on the nomenclature for the cellulose synthase genes in Populus. Trends Plant Sci. 2009, 14, 248-254.

24. Suzuki, S.; Li, L.; Sun, Y.H.; Chiang, V.L. The cellulose synthase gene superfamily and biochemical functions of xylem-specific cellulose synthaselike genes in Populus trichocarpa. Plant Physiol. 2006, 142, 1233-1245.

25. Ranik, M.; Myburg, A.A. Six new cellulose synthase genes from Eucalyptus are associated with primary and secondary cell wall biosynthesis. Tree Physiol. 2006, 26, 545-556.

26. Nairn, C.J.; Haselkorn, T. Three loblolly pine CesA genes expressed in developing xylem are orthologous to secondary cell wall CesA genes of angiosperms. New Phytol. 2005, 166, 907-915.

27. Palle, S.R.; Candace, M.S.; Seeve, C.M.; Eckert, A.J.; Cumbie, W.P.; Goldfarb, B.; Loopstra, C.A. Natural variation in expression of genes involved in xylem development in loblolly pine (Pinus taeda L.) Tree Genet. Genomes 2011, 7, 193-206.

28. Djerbi, S.; Lindskog, M.; Arvestad, L. The genome sequence of black cottonwood (Populus trichocarpa) reveals 18 conserved cellulose synthase (CesA) genes. Planta 2005, 221, 739-746.

29. Tuskan, G.A.; Difazio, S.; Jansson, S.; Bohlmann, J.; Grigoriev, I.; Hellsten, U.; Putnam, N.; Ralph, S.; Rombauts, S.; Salamov, A.; et al. The genome of black cottonwood; Populus trichocarpa (Torr. \& Gray). Science 2006, 313, 1596-1604.

30. Dai, C.; Liu, X.M.; Zhou, F. Selection of interal control genes in semi-quantitative RT-PCR in Betula platyphylla. Nonwood For. Res. 2011, 29, 34-39.

31. Roberts, A.W.; Bushoven, J.T. The cellulose synthase (CESA) gene superfamily of the moss Physcomitrella patens. Plant Mol. Biol. 2007, 63, 207-219.

32. Beeckman, T.; Przemeck, G.K.H.; Stamatiou, G.; Lau, R.; Terryn, N.; De Rycke, R.; Inzé, D.; Berleth, T. Genetic complexity of cellulose synthase a gene function in Arabidopsis embryogenesis. Plant Physiol. 2002, 130, 1883-1893.

33. Gillmor, C.S.; Poindexter, P.; Lorieau, J.; Palcic, M.M.; Somerville, C. $\alpha$-Glucosidase I is required for cellulose biosynthesis and morphogenesis in Arabidopsis. J. Cell Biol. 2002, 156, $1003-1013$. 
34. Hamann, T.; Osborne, E.; Youngs, H.; Misson, J.; Nussaume, L.; Somerville, C. Global expression analysis of CESA and CSL genes in Arabidopsis. Cellulose 2004, 11, 279-286.

35. Brown, R.M.; Zeef, LAH.; Ellis, J.; Goodacre, R.; Turner, S.R. Identification of novel genes in Arabidopsis involved in secondary cell wall formation using expression profiling and reverse genetics. Plant Cell 2005, 17, 2281-2295.

36. Arioli, T.; Peng, L.; Betzner, A.S.; Burn, J.; Wittke, W.; Herth, W.; Camilleri, C.; Höfte, H.; Plazinski, J.; Birch, R.; et al. Molecular analysis of cellulose biosynthesis in Arabidopsis. Science 1998, 279, 717-720.

37. Stork, J.; Harris, D.; Griffiths, J.; Williams, B.; Beisson, F.; Li-Beisson, Y.; Mendu, V.; Haughn, G.; Debolt, S. CELLULOSE SYNTHASE9 serves a nonredundant role in secondary cell wall synthesis in Arabidopsis epidermal testa cells. Plant Physiol. 2010, 153, 580-589.

38. Willats, W.G.T.; McCartney, L.; Mackie, W.; Knox, J.P. Pectin: Cell biology and prospects for functional analysis. Plant Mol. Biol. 2001, 47, 9-27.

39. Young, R.E.; McFarlane, H.E.; Hahn, M.G.; Western, T.L.; Haughn, G.W.; Samuels, A.L. Analysis of the Golgi apparatus in Arabidopsis seed coat cells during polarized secretion of pectin-rich mucilage. Plant Cell 2008, 20, 1623-1638.

40. Mendu, V.; Griffiths, J.S.; Persson, S.; Stork, J.; Downie, A.B.; Voiniciuc, C.; Haughn, G.W.; DeBolt, S. Subfunctionalization of cellulose synthases in seed coat epidermal cells mediates secondary radial wall synthesis and mucilage attachment. Plant Physiol. 2011, 157, 441-453.

41. ORF Finder. Available online: http://www.ncbi.nlm.nih.gov/gorf/orfig.cgi/ (access on 20 September 2012).

42. Allex, C.F. Computational Methods for Fast and Accurate DNA Fragment Assembly. Ph.D. Thesis, University of Wisconsin-Madison, 1999.

43. Hall, T.A. BioEdit: A user-friendly biological sequence alignment editor and analysis program for Windows 95/98/NT. Nucl. Acids. Symp. Ser 1999, 41, 95-98.

44. Tamura, K.; Dudley, J.; Nei, M.; Kumar, S. MEGA4: Molecular evolutionary genetics analysis (MEGA) software version 4.0. Mol. Biol. Evol. 2007, 24, 1596-1599.

45. TMpred-Prediction of transmembrane refions and orientation. Available online: http://www.ch.embnet.org/software/TMPRED_form.html (access on 20 September 2012).

46. Grigoriev, I.V.; Nordberg, H.; Shabalv, I.; Aerts, A.; Cantor, M.; Goodstein, D.; Kuo, A.; Minovitsky, S.; Nikitin, R.; Ohm, R.A. The genome protal of the department of energy joint genome institute. Nucleic. Acids Res. 2011, doi: 10.1093/nar/gkr947.

47. Search for Conserved Domains within a protein or coding nucleotide sequence. Available online: http://www.ncbi.nlm.nih.gov/Structure/cdd/wrpsb.cgi (access on 20 September 2012).

48. Marchler-Bauer, A.; Lu, S.; Anderson, J.B.; Chitsaz, F.; Derbyshire, M.K.; DeWeese-Scott, C.; Fong, J.H.; Geer, L.Y.; Geer, R.C.; Gonzales, N.R.; et al. CDD: A conserved domain database for the functional annotation of proteins. Nucleic Acids Res. 2010, 24, 1-5.

49. Liang, X.; Joshi, C.P. Molecular cloning of ten distinct hypervariable regions from the cellulo se synthase gene superfamily in aspen trees. Tree Physiol. 2004, 24, 543-550.

50. Vergara, C.E.; Carpita, N.C. $\beta$-D-Glycan synthases and the CesA gene family: Lessons to be learned from the mixed-linkage $(1 \rightarrow 3) ;(1 \rightarrow 4) \beta$-D-glucan synthase. Plant Mol. Biol. 2001, 47, $145-160$. 
51. Chen, P.F.; Liu, X.M.; Song, F.N.; Song, X.S.; Liu, N.; Jin, W.W.; Liu, W. Cloning and sequence analysis of full-length cDNA of actin gene from Birch (Betula platyphylla Suk.). Bull. Bot. Res. 2009, 29, 339-345.

52. Livak, K.J.; Schmittgen, T.D. Analysis of relative gene expression data using real-time quantitative PCR and the 2(-delta delta C(T)) method. Methods 2001, 25, 402-408.

(C) 2012 by the authors; licensee MDPI; Basel; Switzerland. This article is an open access article distributed under the terms and conditions of the Creative Commons Attribution license (http://creativecommons.org/licenses/by/3.0/) 